\section{Case Reports in Ophthalmology}

Case Rep Ophthalmol 2020;11:402-410

DOI: 10.1159/000508912

Published online: August 5, 2020
(C) 2020 The Author(s)

Published by S. Karger AG, Basel www.karger.com/cop

\title{
Intravitreal Voriconazole for Treatment of Bilateral Endogenous Candida Chorioretinitis
}

\author{
Ha Eun Sim Min Ji Kang Jae Suk Kim Je Hyung Hwang \\ Department of Ophthalmology, Sanggye Paik Hospital, Inje University of Korea College of \\ Medicine, Seoul, Republic of Korea
}

\section{Keywords}

Candida chorioretinitis · Endogenous chorioretinitis · Voriconazole

\begin{abstract}
This report describes a patient with bilateral endogenous candida chorioretinitis. The patient had a 2-day history of bilateral blurred vision. Fundus photography revealed multiple chorioretinal infiltrations in both eyes and a parafoveal hemorrhage in the left eye. After 2 days, fundus examination showed an increased number of infiltrations and hemorrhages in both eyes and worsening vitreous inflammation. A large infiltrative intraretinal lesion and a retinal hemorrhage of the left eye were discovered on optical coherence tomography. Candida albicans was diagnosed from blood culture. The bilateral candida chorioretinitis had not responded to systemic or topical antifungal medication. The chorioretinitis was refractory to intravitreal amphotericin B as well. Intravitreal voriconazole injection in both eyes and intravitreal bevacizumab injection in the left eye were performed thereafter. The chorioretinal infiltrations and hemorrhages decreased in both eyes. Intravitreal voriconazole injection was effective in the treatment of intractable candida chorioretinitis.

(C) 2020 The Author(s)

Published by S. Karger AG, Basel
\end{abstract}




\section{Case Reports in Ophthalmology}

\section{Introduction}

Immunocompromised patients are at increased risk of fungal sepsis. Fungal dissemination to the organs occurs via hematogenous seeding and may include the retinal and choroidal capillaries in the eye. Candida species are the most common cause of endogenous fungal endophthalmitis [1]. A previous study described two main types of ocular candidiasis: endogenous endophthalmitis, which is characterized by vitritis and fluffy balls extending into the vitreous body, and candida chorioretinitis, in which the disease is restricted to the chorioretinal layer [2]. Ocular candidiasis can be treated by systemic or intravitreal injection of an antifungal agent. Voriconazole is a broad-spectrum antifungal agent that is derived from fluconazole and has been shown to achieve therapeutically significant concentrations in the vitreous body after oral administration [3]. Here we report a case of candida chorioretinitis that was unresponsive to oral voriconazole and intravitreal amphotericin B.

\section{Case Presentation}

A 62-year-old Korean woman was referred to our clinic with a 2-day history of bilateral blurred vision. She was receiving chemotherapy for anorectal cancer in the internal medicine department and intravenous fluconazole $(10 \mathrm{mg} / \mathrm{kg}$ i.v. every $24 \mathrm{~h} \times 24$ days $)$ to treat candidemia. Candida albicans was diagnosed from blood culture. Meningitis was not observed on neurological examination. Her past ophthalmic history was unremarkable.

On examination, her best-corrected distance visual acuity was 20/40 in the right eye and $20 / 200$ in the left eye. Slit-lamp examination of the cornea and conjunctiva was unremarkable, and there was no active inflammation in the anterior chamber or neovascularization in the iris. Intraocular pressure was normal in both eyes. Fundus photography revealed multiple chorioretinal infiltrations in both eyes and a parafoveal hemorrhage in the left eye (Fig. 1). The patient's general condition was considered to be very poor, so we could not perform optical coherence tomography or fundus fluorescein angiography. Given the good intravitreal penetration of voriconazole [3], oral voriconazole was added to treat the candida chorioretinitis (400 mg every $12 \mathrm{~h} \times 21$ days). After 2 days of treatment with oral voriconazole, fundus examination showed an increased number of infiltrations and hemorrhages in both eyes and worsening vitreous inflammation. The parafoveal hemorrhage was increased in the left eye and the macula appeared to be elevated. The patient's general condition then deteriorated further and she was no longer able to visit our clinic. Therefore, a fundus examination was performed and bilateral intravitreal amphotericin B injections $0.1 \mathrm{~mL}(50 \mu \mathrm{g} / \mathrm{mL})$ were administered while she was an inpatient in the internal medicine ward. Four days after the intravitreal injections, the patient's visual acuity decreased; at this time, her best-corrected distance visual acuity was $20 / 60$ in the right eye and $2 / 100$ in the left eye. On fundus examination, there were further infiltrations and hemorrhages in both eyes. The patient's general condition was improved (body temperature had normalized and WBC and inflammatory marker levels [ESR, CRP] had decreased on the blood tests), so we were able to perform optical coherence tomography, which revealed parafoveal hemorrhage in the left eye (Fig. 2). We administered intravitreal voriconazole injections $0.1 \mathrm{~mL}(100 \mu \mathrm{g} / 0.1 \mathrm{~mL})$ bilaterally in the left eye. One week later, the chorioretinal infiltrations and hemorrhages were decreased in both eyes (Fig. 3). At the 1- and 3-month follow-up visits there was no recurrence of chorioretinitis (Fig. 4, 5). 


\section{Case Reports in Ophthalmology}

\section{Discussion and Conclusion}

The risk factors for candidemia include long-term intravenous therapy, gastrointestinal surgery, trauma, indwelling bladder catheter, immunocompromised status, malignancy, and intravenous drug abuse. Our patient had three of these risk factors in the form of anorectal cancer, chemotherapy, and long-term intravenous therapy. For chemotherapy, a chemoport placed on October 31, 2017 via the right jugular vein was used; the patient received fourthline chemotherapy with bevacizumab, 5 -fluorouracil, leucovorin, and oxaliplatin. During follow-up, the patient developed fever and was thus hospitalized for fifth-line chemotherapy with bevacizumab, 5 -fluorouracil, leucovorin, and oxaliplatin and the evaluation of fever. During hospitalization, candida bacteremia was confirmed in blood culture. Hence, the chemoport was removed and a peripherally inserted central catheter was inserted through the right basilic vein. Chorioretinitis has been reported to occur in $7.9-37 \%$ of patients with candidemia [4].

Feman et al. [5] reported that inpatients were less likely to develop ocular candidiasis because of early diagnosis of candidemia and prompt systemic antifungal therapy. However, in our patient, early diagnosis of candidemia and intravenous fluconazole therapy did not prevent ocular candidiasis.

Intravenous amphotericin has been recommended as a treatment for fluconazole-resistant C. albicans, C. krusei, and C. glabrata [6]. However, systemic amphotericin is not suitable for immunocompromised patients because of its poor intravitreal penetration and systemic side effects [7]. In our patient, intravenous fluconazole was effective for systemic candidemia and systemic amphotericin could not be used because of the patient's poor overall condition. Voriconazole is a broad-spectrum second-generation derivative of fluconazole that has $96 \%$ bioavailability and has been shown to reach therapeutically significant concentrations in the vitreous body after oral administration [3]. Biju et al. [1] reported that candida endophthalmitis could be treated successfully by oral administration of voriconazole, but this strategy was not effective in our patient. Intravitreal liposomal amphotericin B injection has been reported to be an effective treatment for endogenous candida endophthalmitis [8]. Voriconazole is cleared from the vitreous cavity within $24 \mathrm{~h}$ of injection, so multiple intravitreal injections are required to maintain therapeutic concentrations in the eye [8].

Considering the patient's generally poor condition, we opted to administer intravitreal amphotericin B instead of voriconazole to avoid the need for multiple intravitreal injections. In South Korea, voriconazole is only indicated in fungal infections that do not respond to amphotericin B or fluconazole. However, intravitreal amphotericin was not effective in our patient, whereas voriconazole was effective and did not require multiple injections.

Furthermore, the peak, trough, and random serum levels of antifungal agents should be evaluated when using intravenous fluconazole. However, the patient was not evaluated for serum levels of the antifungal agents used because of issues related to medical insurance coverage and expenses, and her condition was clinically (fever, decreased WBC, decreased ESR and CRP) determined in the internal medicine department; this was a limitation of our study.

To our knowledge, this is the first report of endogenous candida chorioretinitis being treated successfully with a single intravitreal injection of voriconazole without vitrectomy.

In conclusion, intravitreal voriconazole could be considered as a first-line treatment for endogenous candida chorioretinitis, despite its short half-life in the vitreous cavity, in immunocompromised patients who do not respond to oral voriconazole or intravitreal amphoteri$\operatorname{cin} \mathrm{B}$. 


\section{Case Reports in Ophthalmology}

Sim et al.: Intravitreal Voriconazole Injection

\section{Statement of Ethics}

Written informed consent for publication of this case report, including publication of images, was obtained from the patient.

\section{Conflict of Interest Statement}

The authors have no conflict of interest to declare.

\section{Funding Sources}

This research received no specific grant from any funding agency in the public, commercial, or not-for-profit sectors.

\section{Author Contributions}

Substantial contributions to the conception or design of the work: H.E. Sim and J.H. Hwang. Acquisition of data: M.J. Kang and J.S. Kim. Drafting of the work: H.E. Sim and J.H. Hwang.

\section{References}

1 Biju R, Sushil D, Georgy NK. Successful management of presumed Candida endogenous endophthalmitis with oral voriconazole. Indian J Ophthalmol. 2009 Jul-Aug;57(4):306-8.

2 Rodríguez-Adrián LJ, King RT, Tamayo-Derat LG, Miller JW, Garcia CA, Rex JH. Retinal lesions as clues to disseminated bacterial and candidal infections: frequency, natural history, and etiology. Medicine (Baltimore). 2003 May;82(3):187-202.

3 Hariprasad SM, Mieler WF, Holz ER, Gao H, Kim JE, Chi J, et al. Determination of vitreous, aqueous, and plasma concentration of orally administered voriconazole in humans. Arch Ophthalmol. 2004 Jan;122(1): 42-7.

4 Parke DW 2nd, Jones DB, Gentry LO. Endogenous endophthalmitis among patients with candidemia. Ophthalmology. 1982 Jul;89(7):789-96.

5 Feman SS, Nichols JC, Chung SM, Theobald TA. Endophthalmitis in patients with disseminated fungal disease. Trans Am Ophthalmol Soc. 2002;100:67-70.

6 Pappas PG, Rex JH, Sobel JD, Filler SG, Dismukes WE, Walsh TJ, et al.; Infectious Diseases Society of America. Guidelines for treatment of candidiasis. Clin Infect Dis. 2004 Jan;38(2):161-89.

7 O’Day DM, Head WS, Robinson RD, Stern WH, Freeman JM. Intraocular penetration of systemically administered antifungal agents. Curr Eye Res. 1985 Feb;4(2):131-4.

8 Bae JH, Lee SC. Intravitreal liposomal amphotericin B for treatment of endogenous candida endophthalmitis. Jpn J Ophthalmol. 2015 Sep;59(5):346-52. 
Case Reports in Ophthalmology
Case Rep Ophthalmol 2020;11:402-410 DOI: $10.1159 / 000508912$

(c) 2020 The www.karger.com/cop

Sim et al.: Intravitreal Voriconazole Injection
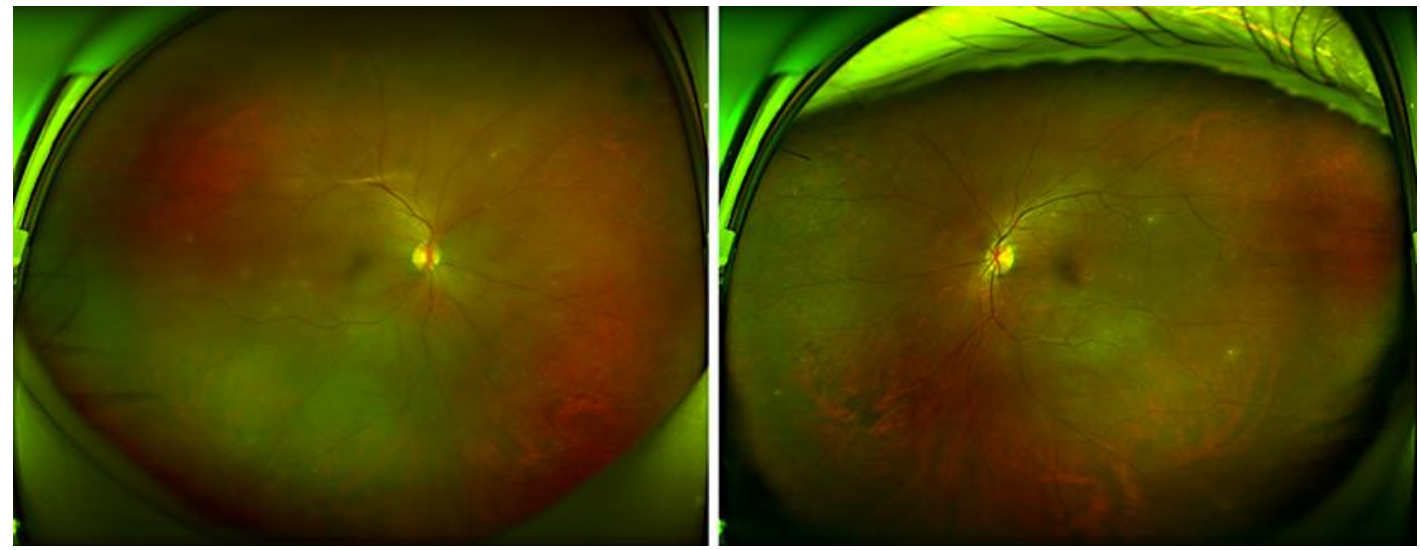

Fig. 1. Fundus photographs of the patient with multiple chorioretinal infiltrations in both eyes and a parafoveal hemorrhage in the left eye. 
Case Reports in Ophthalmology
Case Rep Ophthalmol 2020;11:402-410 DOI: $10.1159 / 000508912$

(c) 2020 The Author(s). Published by S. Karger AG, Basel www.karger.com/cop

Sim et al.: Intravitreal Voriconazole Injection

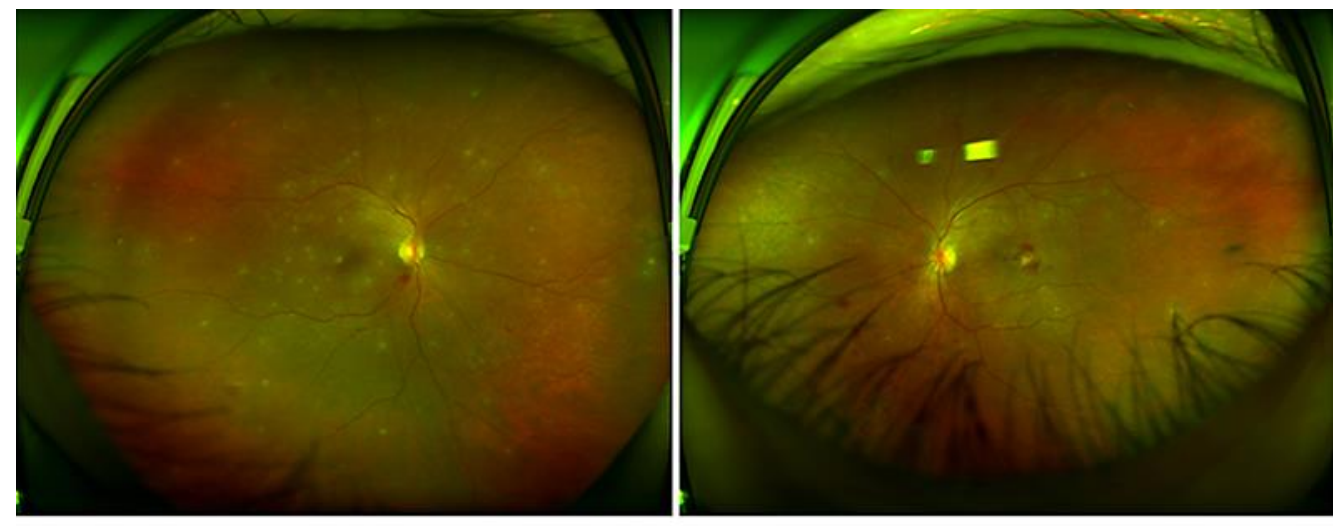

IR 30?ART + OCT 30?(8.8 mm) ART (59) Q: 33 [HS]

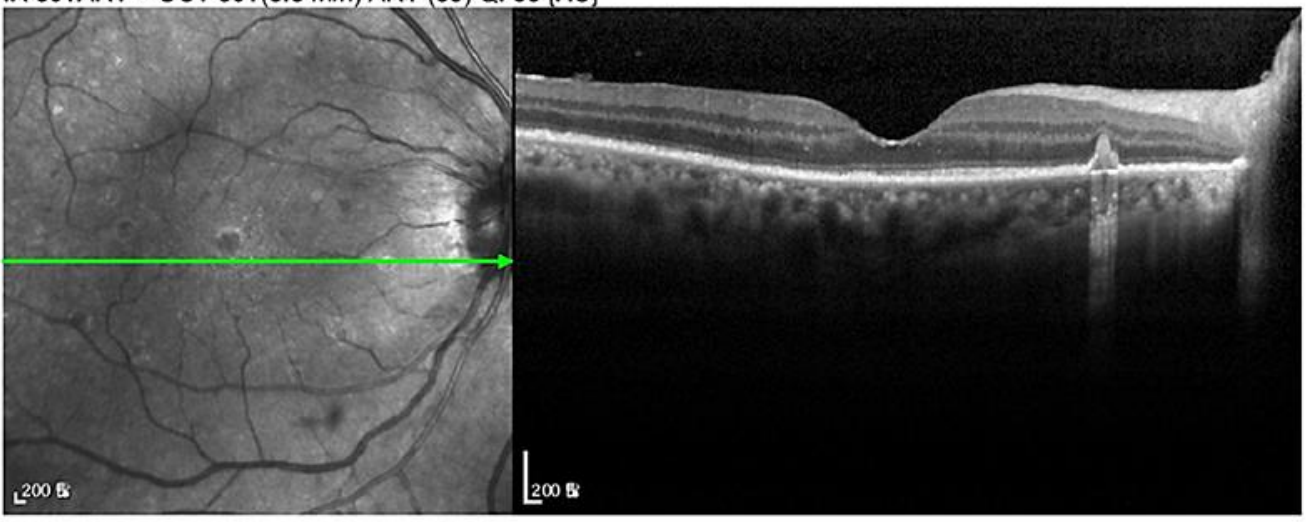

IR 30?ART + OCT 30?(8.6 mm) ART (52) Q: 31 [HS]

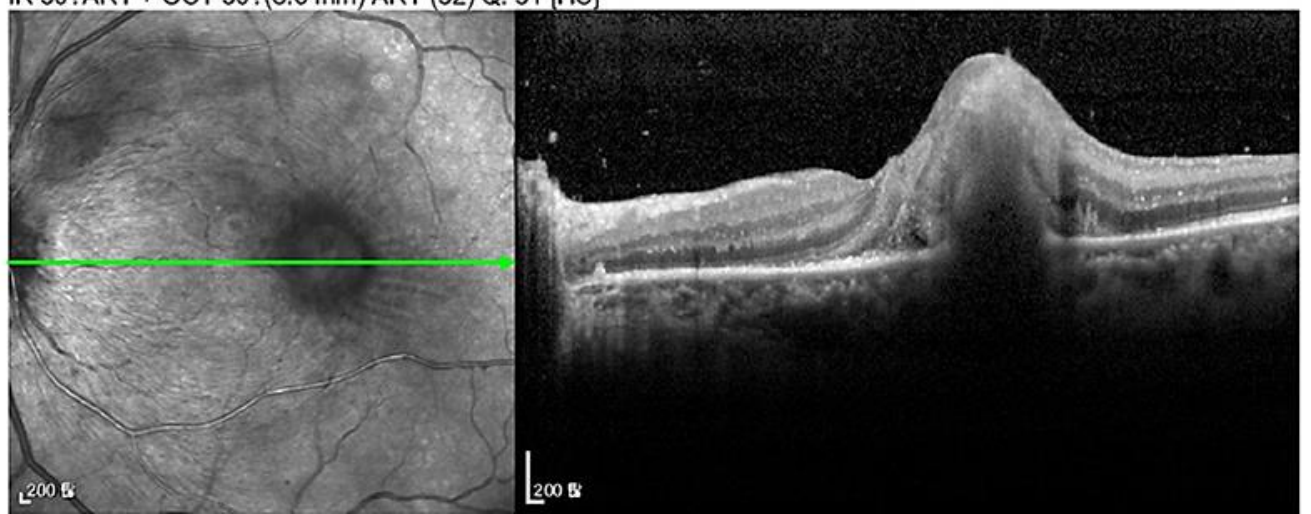

Fig. 2. Fundus photographs and optical coherence tomograms obtained 4 days after intravitreal injection of amphotericin B. There were increased multiple infiltrations and hemorrhages in both eyes. 


\section{Case Reports in Ophthalmology}
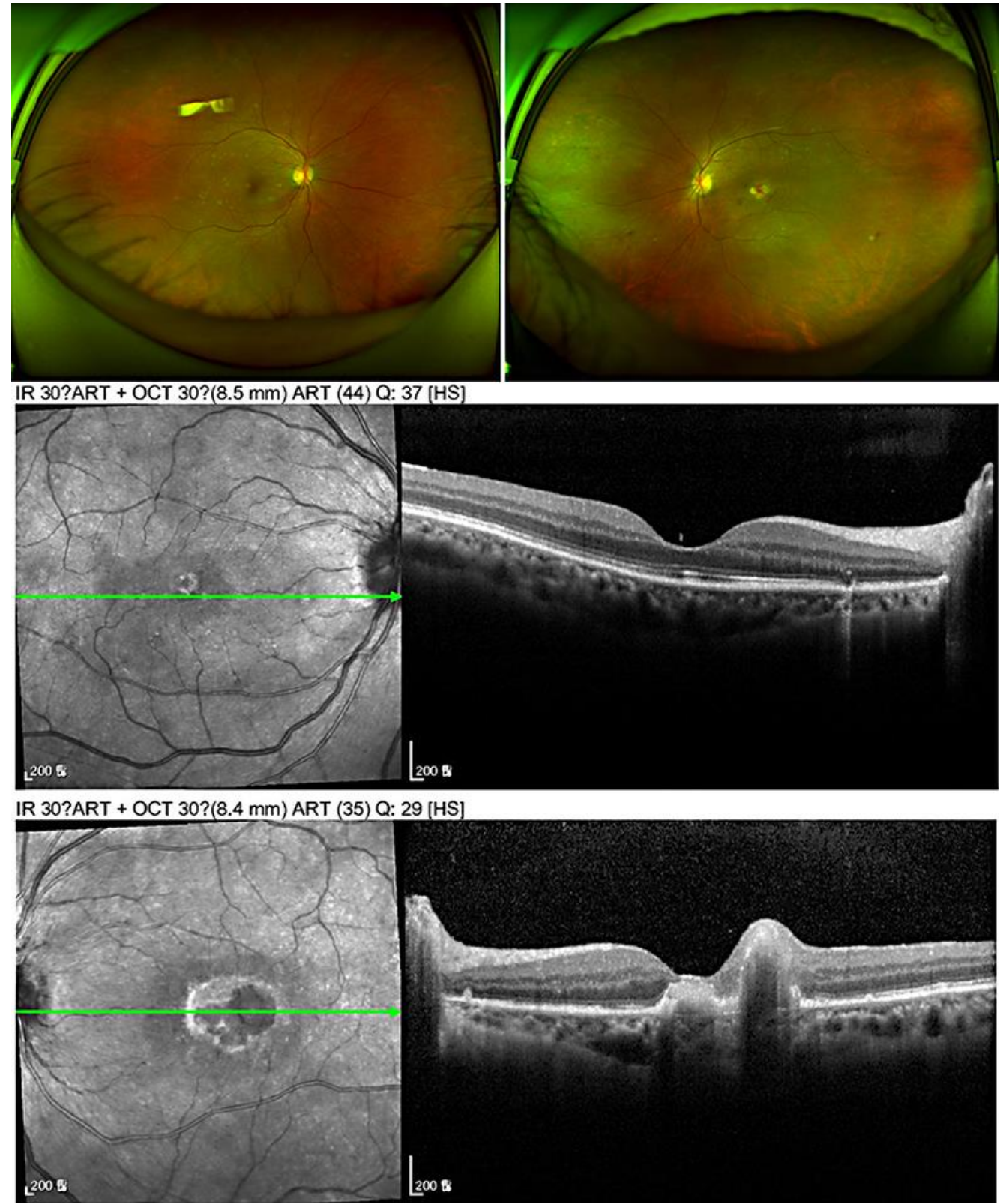

Fig. 3. Fundus photographs and optical coherence tomograms obtained 1 week after bilateral intravitreal injections of voriconazole. There was a decrease in the chorioretinal infiltrations and hemorrhages in both eyes. 
Case Reports in Ophthalmology
Case Rep Ophthalmol 2020;11:402-410

DOI: $10.1159 / 000508912$

(c) 2020 The Author(s). Published by S. Karger AG, Basel www.karger.com/cop

Sim et al.: Intravitreal Voriconazole Injection

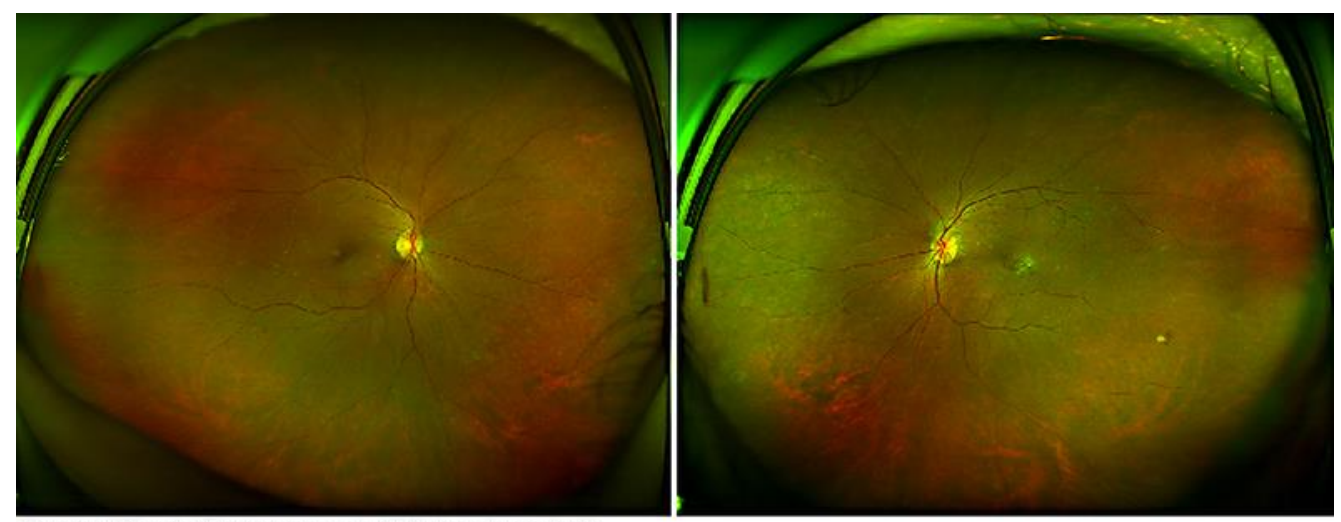

IR $30^{\circ} \mathrm{ART}+\mathrm{OC}^{-} 30^{\circ}(8.5 \mathrm{~mm}) \mathrm{ART}(45) \mathrm{Q}$ 3 [ [-S]

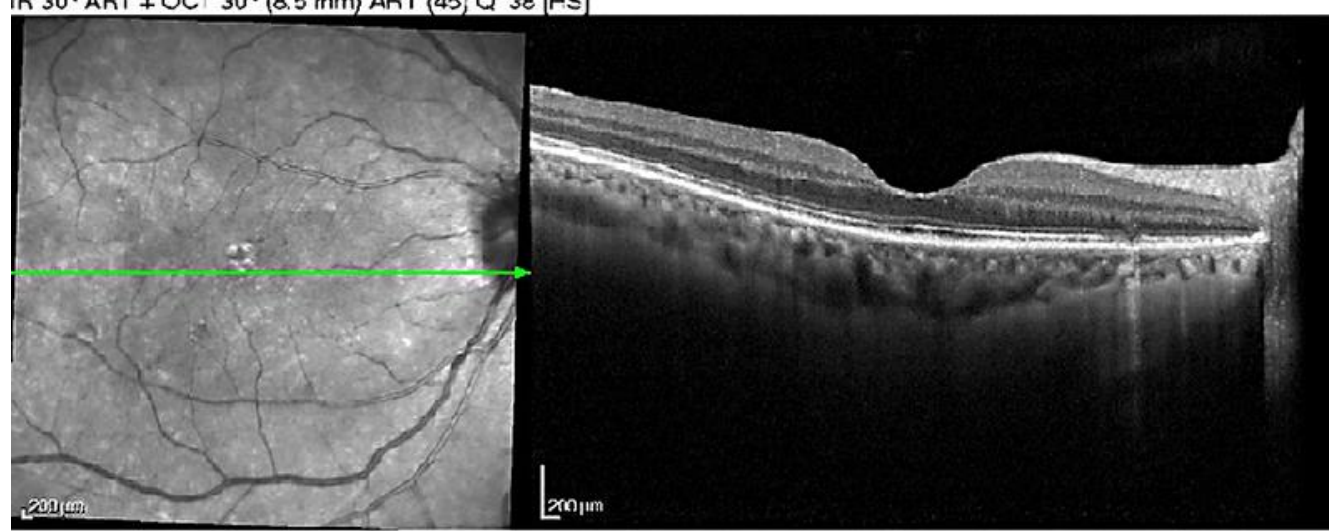

IR $30^{\circ} \mathrm{ART}, \mathrm{OC}^{-} 30^{\circ}(8.5 \mathrm{~mm})$ ART (52) Q 15 [HS]

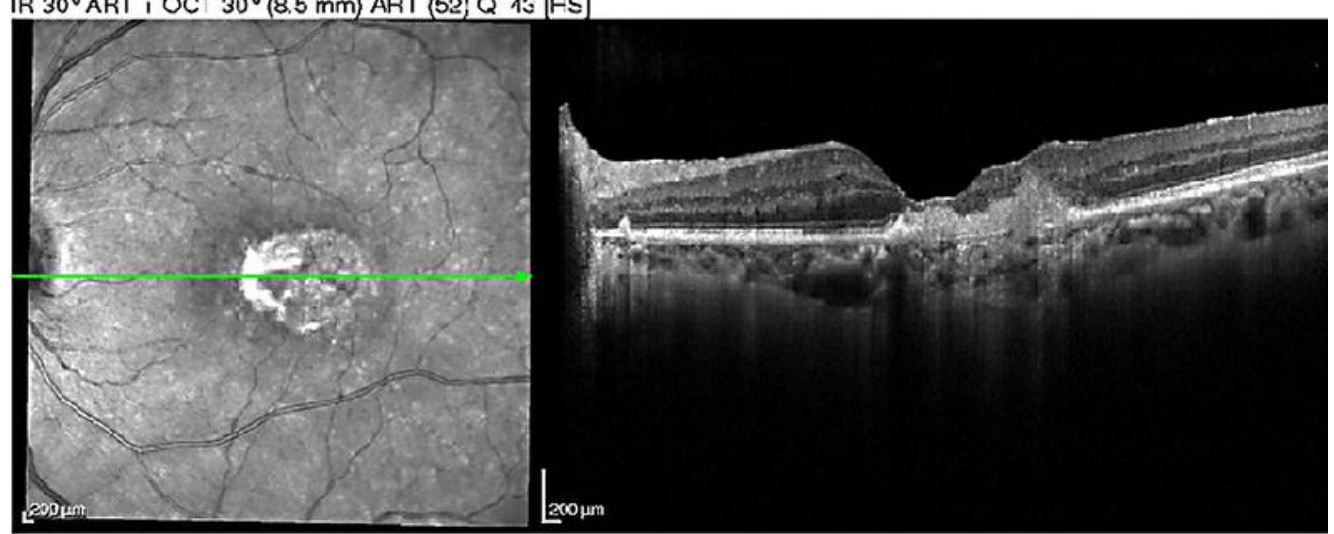

Fig. 4. Fundus photographs and optical coherence tomograms obtained 1 moth after bilateral intravitreal injections of voriconazole. 
Case Reports in Ophthalmology
Case Rep Ophthalmol 2020;11:402-410

DOI: 10.1159/000508912

(C) 2020 The Author(s). Published by S. Karger AG, Basel www.karger.com/cop

Sim et al.: Intravitreal Voriconazole Injection
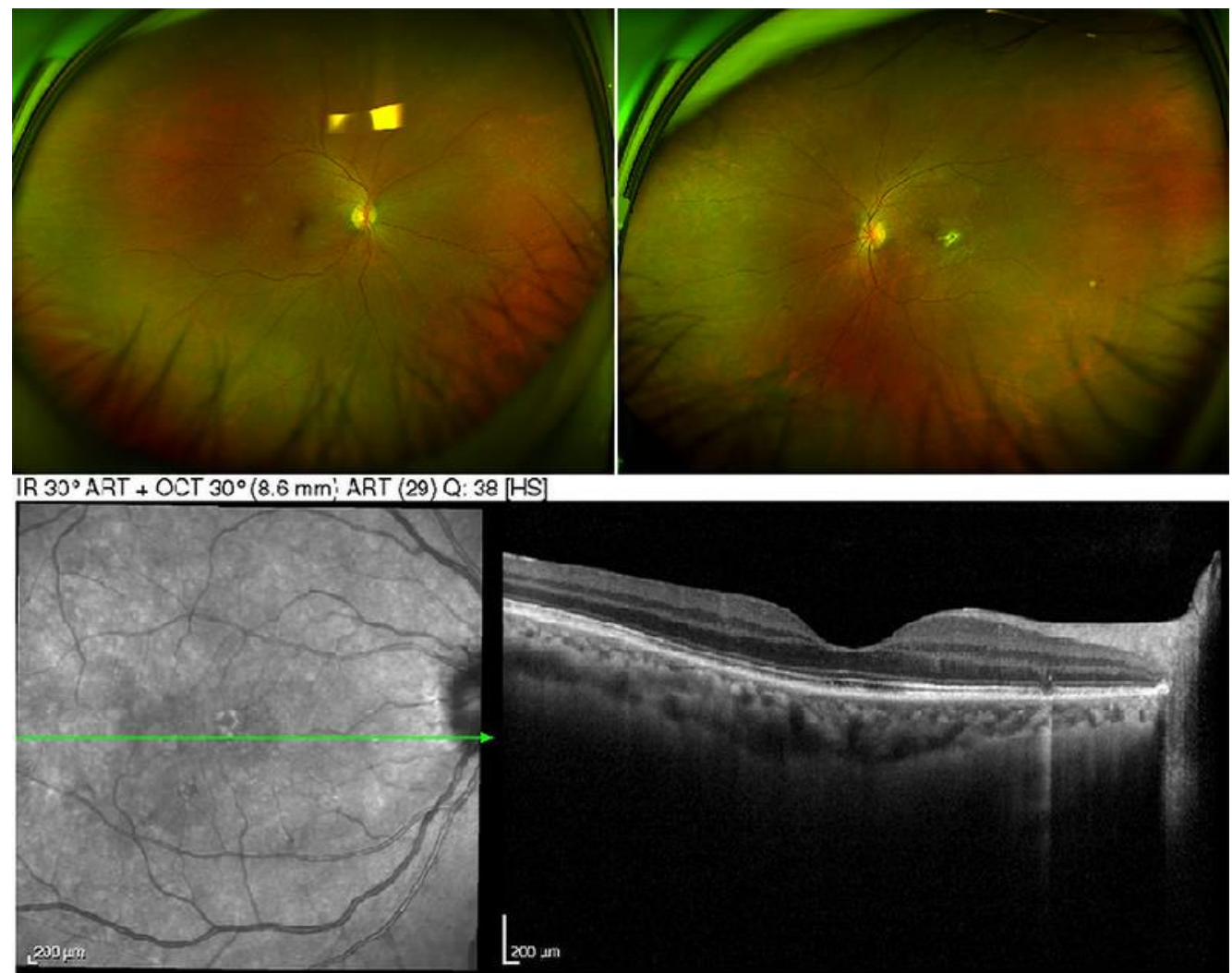

IR $30^{\circ} \mathrm{ART}, \mathrm{OCT} 30^{\circ}(8.6 \mathrm{~mm})$ ART (17) Q: 39 [-IS]

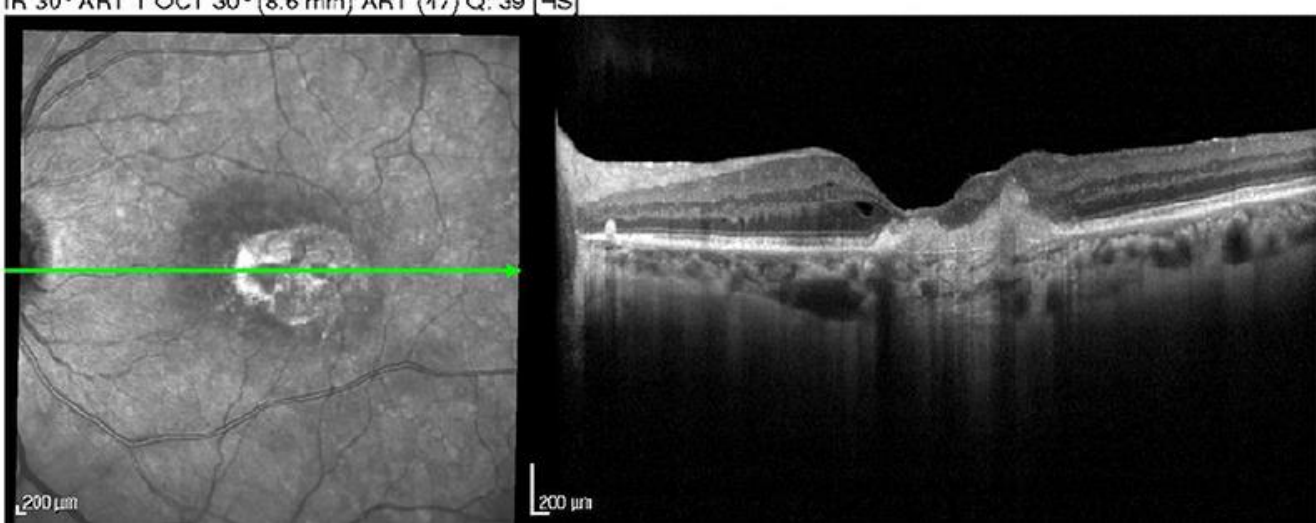

Fig. 5. Fundus photographs and optical coherence tomograms obtained 3 months after bilateral intravitreal injections of voriconazole. 